\title{
Agglomeration of As Antisites in As-Rich Low-Temperature GaAs: Nucleation without a Critical Nucleus Size
}

\author{
T.E. M. Staab* \\ Helmholtz Institut für Strahlen- und Kernphysik, Universität Bonn, Nussallee 14-16, D-53115 Bonn, Germany \\ R. M. Nieminen \\ Laboratory of Physics, Helsinki University of Technology, P.O. Box 1100, FIN-02015 HUT, Finland \\ M. Luysberg \\ Institut für Festkörperforschung, Forschungszentrum Jülich, D-52425 Jülich, Germany \\ Th. Frauenheim \\ Department of Physics, Theoretical Physics, University GH Paderborn, D-33098 Paderborn, Germany
}

(Received 16 March 2005; published 14 September 2005)

\begin{abstract}
To investigate the early stages of nucleation and growth of As precipitates in GaAs grown at low substrate temperature, we make use of a self-consistent-charge density-functional based tight-binding method. Since a pair of As antisites already shows a significant binding energy which increases when more As antisites are attached, there is no critical nucleus size. Provided that all excess As has precipitated, the clusters may grow in size since the binding energies increase with increasing agglomeration size. These findings close the gap between experimental investigation of point defects and the detection of nanometer-size precipitates in transmission electron microscopy.
\end{abstract}

DOI: 10.1103/PhysRevLett.95.125502

Starting from a supersaturated solution, any system will finally end up in equilibrium separated into two phases. Nevertheless, the initial process of nucleation and the very first stages of growth towards the formation of precipitates remain poorly understood, since experimentally it is very difficult to access the microscopic structure of the early stages of precipitation. Two models describe the precipitation process: spinodal decomposition and nucleation/ growth. Spinodal decomposition is distinguished from nucleation by the observation that in the latter case the forming nucleus has already in the very early stage the correct concentration of the final precipitation, while in the former case the concentration increases gradually (uphill diffusion).

GaAs grown at a low substrate temperature of $200^{\circ} \mathrm{C}$ (LT GaAs) is the perfect model system for studying the process of nucleation and growth. LT GaAs is a special system, since the density of grown-in defects is very high compared to semiconductors in general. The material contains up to $1 \%$ excess As, which is incorporated in the form of point defects: $\approx 10^{20} \mathrm{~cm}^{-3}$ neutral As antisite $\mathrm{As}_{\mathrm{Ga}}^{0}[1]$, $\approx 10^{18} \mathrm{~cm}^{-3}$ negatively charge $\mathrm{Ga}$ vacancies $\mathrm{V}_{\mathrm{Ga}}^{3-}$, and $\approx 3 \times 10^{18} \mathrm{~cm}^{-3}$ positively charge As antisites As $\mathrm{Aa}^{+}$ $[2,3]$. The large density of defects causes a tetragonal lattice strain in the growth direction of up to $0.16 \%$.

Nevertheless, LT GaAs is a perfectly simple system: only intrinsic defects exist if no doping atoms have been added. During annealing at temperatures higher than $400{ }^{\circ} \mathrm{C}$, the initial high point defect concentration decreases by 2 orders of magnitude, while the lattice distortion simultaneously vanishes $[4,5]$. The existing excess As is found to precipitate and form small clusters of pure
PACS numbers: $61.72 . \mathrm{Bb}, 61.72 . \mathrm{Ji}, 71.15 . \mathrm{Mb}$ arsenic [4,6]. Unfortunately, As precipitates become visible in the transmission electron microscope (TEM) first when they have reached a diameter of more than $1 \mathrm{~nm}$ [4], and consist of several tens of As atoms. While the point defects have been characterized prior to the precipitation process by optical and EPR [3-5] as well as positron annihilation methods $[7,8]$, the early stage of precipitation is hardly accessible experimentally.

For larger precipitate sizes their coarsening can be described in LT GaAs by diffusion-limited Ostwald ripening with an activation energy of $\approx 1.4 \mathrm{eV}$ [9]. This is also the calculated migration enthalpy for the Ga vacancy within an error margin of $0.1 \mathrm{eV}[10,11]$. Thus grown-in gallium vacancies are suspected to act as diffusion vehicles [12].

Since the As antisite is known to strain the lattice (outward relaxation of nearest-neighbor As atoms on lattice sites [13-15]), it is not evident a priori that two neighboring As antisites are bound together. Pairing them is expected to strain the lattice locally even more so than two widely separated antisites. Hence, one might expect that this pair is unstable and just dissociates. The question arises then of how the nucleation and growth of the nuclei start on the microscopic level. The probability that diffusing species randomly form a critical nucleus consisting of at least three atoms is only $2.7 \times 10^{-5}$ or $0.0027 \%$ - as elementary probability theory shows.

Important questions deal with the size of the nuclei formed, how the As clusters grow, and if there are certain configurations especially stable against dissociation. It will turn out that the critical nucleus size is just a pair of As antisites, since they already bind. 
By looking at the energetics on an atomic level in this Letter, we close the gap between experimentally observable isolated point defects and larger agglomerates in the nanometer region (visible in TEM). Hence, we study the stability of small As agglomerates up to 17 As antisites involved, which corresponds to a diameter of the As precipitate of nearly $1.5 \mathrm{~nm}$. In contrast to most textbooks on metal physics, where precipitations are restricted to spherical shape and the distortions of the lattice are disregarded (see, e.g., [16]), our calculations consider also nonspherical particles and elastic distortions are included automatically.

The lattice distortions and formation energies for $\mathrm{As}_{\mathrm{Ga}}$ antisites and their agglomerates are calculated by using a self-consistent-charge density-functional based tightbinding (SCC-DFTB) method [17]. This method is capable of efficiently treating large supercells with 512 atoms, hence allowing one to treat nanometer-sized precipitates and thus enabling direct comparison with experiments.

The method employs a basis of numerically derived $s$ and $p$ confined atomic orbitals which are obtained within the self-consistent field (SCF) local-density approximation (LDA). All two-center integrals of the density-functional theory Hamiltonian and overlap matrix are evaluated explicitly. Charge transfer is taken into account through the incorporation of self-consistency for the distribution of the Mulliken charges. This is based on a second order expansion of the Kohn-Sham energy. For a detailed description of the SCC-DFTB method and its application to GaAs, see Refs. [17,18].

Recent results, including calculations for $\mathrm{As}_{\mathrm{Ga}}$ antisites and extended defects, have demonstrated the validity of the SCC-DFTB method for modeling defects in GaAs [19]. These calculations are in good agreement with full $a b$ initio SCF-LDA results [20]. In contrast to more sophisticated methods, the computing time and memory usage of SCC-DFTB is up to 2 orders of magnitude smaller. All calculations were done within the $\Gamma$-point approximation. The numerical error in total energy is less than $0.005 \mathrm{eV}$, indicating well converged results.

Although defects in GaAs can be charged, we do not consider such cases and restrict the calculations to neutral As antisites and their agglomerates (corresponding to charge-neutral supercells). This is justified by the fact that the concentration of the neutral $\mathrm{As}_{\mathrm{Ga}}^{0}$ is more than 10 times larger than that of the positive $\mathrm{As}_{\mathrm{Ga}}^{+}$[21]. On the other hand, electrical measurements do not indicate high densities of other electrically active defects.

The first unexpected result is that the smallest possible agglomerate of As antisites on closest sites, the nextneighbor pair on the $\mathrm{Ga}$ sublattice, exhibits a binding energy of about $200 \mathrm{meV}$. The binding of the As-antisite pair is facilitated by the more metallic bonding in the As atom cloud and its associated charge delocalization. Provided that the As-antisite pair should be the primary nucleus for the growth process, attaching more antisites must be energetically favorable.
Hence, we checked all possible configurations of three As antisites on neighboring Ga-lattice sites. The possible arrangements differ in formation energy by more than $400 \mathrm{meV}$. The most unfavorable ones are chains of antisites, agglomerates on the $\{001\}$ plane (the growth plane of LT GaAs) as well as on a single $\{111\}$ plane. But we also find an energetically favorable configuration, shown in Fig. 1, which leads to a formation energy much lower than that of three As antisites separated in the lattice. The energy gain is about $600 \mathrm{meV}$ (see also Table I), and the lattice is only slightly distorted.

Larger agglomerates were constructed by the following principles and in accordance with experimental observations: from a macroscopic viewpoint, one would expect that the lowest energy configuration for a given number of As antisites would be one where the As antisites form the most compact cluster possible, minimizing the surface energy while inducing the smallest possible strain to the lattice. This is also the underlying principle of nucleation and growth, where size and shape are determined by the interplay between the energy gain in phase separation versus the total cost in surface energy and elastic distortion. Additionally, it has been experimentally observed that larger As precipitates have the shape of rounded tetrahedra on $\{111\}$ planes in the GaAs lattice [22].

The smallest compact As cluster, where the four Ga nearest-neighbor atoms of one As lattice atom are replaced by four As antisites, is spherically symmetric and hence

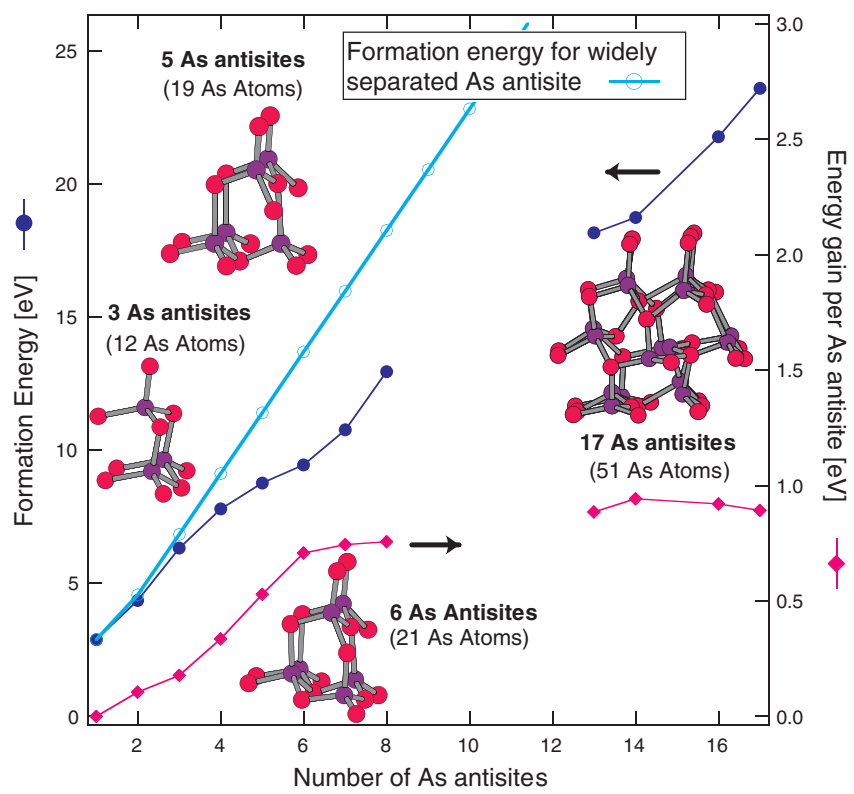

FIG. 1 (color online). Formation energy $E_{\mathrm{F}}$ for As precipitates of different sizes and, for comparison, the sum of the formation energy for separated $\mathrm{As}_{\mathrm{Ga}}$. Also shown is the energy gain $E_{\text {gain }}$ per As antisite due to clustering. The atomic configurations of arsenic agglomerates are projected along a $\langle 111\rangle$ direction. As lattice atoms surrounding the antisites are also shown. Bonds are shown for atomic distances smaller than $330 \mathrm{pm}$. The antisites are shown in a darker color (violet). 
TABLE I. Formation energy $E_{\mathrm{F}}$, energy gain by precipitation $E_{\text {gain }}$, and binding energies $E_{\mathrm{b}}$ for the last $\mathrm{As}_{\mathrm{Ga}}$ in As-rich GaAs dependent on the size of precipitation (number $n$ of $\mathrm{As}_{\mathrm{Ga}}$ and the arrangement of antisites. All antisites are arranged on a number of $\{111\}$ planes as given in the second column having roughly the diameter $d$ (notation explained in the text).

\begin{tabular}{|c|c|c|c|c|c|c|}
\hline$n$ & Plane & $\begin{array}{c}d \\
{[\mathrm{~nm}]}\end{array}$ & $\begin{array}{c}E_{\mathrm{F}} \\
{[\mathrm{eV}]}\end{array}$ & $\begin{array}{l}E_{\text {gain }} \\
\text { total }\end{array}$ & $\begin{array}{c}{[\mathrm{eV}]} \\
\text { per } \mathrm{As}_{\mathrm{Ga}}\end{array}$ & $\begin{array}{r}E_{\mathrm{b}}[\mathrm{eV}] \\
\text { last } \mathrm{As}_{\mathrm{Ga}}\end{array}$ \\
\hline 1 & & $\ldots$ & 2.28 & $\ldots$ & $\ldots$ & $\ldots$ \\
\hline 2 & & $\ldots$ & 4.35 & 0.21 & 0.105 & -0.21 \\
\hline 3 & 210 & $0.65-0.80$ & 6.31 & 0.53 & 0.177 & -0.32 \\
\hline 4 & 220 & $0.70-0.85$ & 7.78 & 1.34 & 0.335 & -0.81 \\
\hline 5 & 320 & $0.79-0.93$ & 8.76 & 2.64 & 0.528 & -1.3 \\
\hline 6 & 420 & $0.80-1.12$ & 9.44 & 4.24 & 0.707 & -1.6 \\
\hline 7 & 520 & $\ldots$ & 10.75 & 5.21 & 0.744 & -1.03 \\
\hline 8 & 530 & $\ldots$ & 12.95 & 5.29 & 0.756 & -0.12 \\
\hline 13 & 373 & $1.05-1.28$ & 18.14 & 11.5 & 0.885 & $\ldots$ \\
\hline 14 & 950 & $1.10-1.30$ & 18.73 & 13.2 & 0.942 & $\cdots$ \\
\hline 16 & 952 & $1.28-1.38$ & 21.76 & 14.7 & 0.920 & $\ldots$ \\
\hline 17 & 575 & $1.25-1.38$ & 23.58 & 15.2 & 0.893 & $\cdots$ \\
\hline
\end{tabular}

has a minimal surface. Considering the 12 As lattice atoms in the next-nearest-neighbor shell as belonging to the cluster (15 As atoms in total), the pure arsenic region extends to a diameter of about $0.85 \mathrm{~nm}$. Although clearly the interface towards the surrounding GaAs crystal is minimized, this is not the case for the total energy. The energy gain per $\mathrm{As}_{\mathrm{Ga}}$ in forming this spherical As cluster accounts for only $0.29 \mathrm{eV}$ compared to $1.34 \mathrm{eV}$ for the most stable configuration for four $\mathrm{As}_{\mathrm{Ga}}$ located on two consecutive $\{111\}$ planes (see also Table I).

In energetically unfavorable configurations, most of the involved As antisites are not able to obtain their favored bonding model similar to bulk arsenic: three short bonds $(\approx 252 \mathrm{pm})$ with bond angles of about $96^{\circ}$ and one long bond $(\approx 312 \mathrm{pm})$ as shown in Fig. 2 .

Further examples for unfavorable agglomerates are As antisites on $\{001\}$ planes. Obviously, there the As antisites can hardly change their bonding configuration due to the restrictions of the surrounding lattice (bond lengths stay at about $275 \mathrm{pm})$. Hence, the total energy of formation is
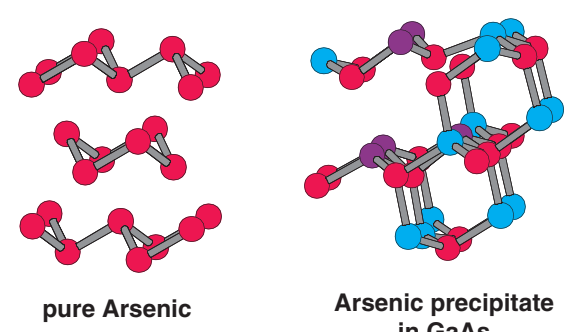

Arsenic precipitate in GaAs

FIG. 2 (color online). Atomic arrangement for bulk As and the energetically most favorable agglomerate of five As antisites looking along a (111) plane. As atoms are dark gray (magenta); Ga atoms are light gray (cyan). The antisites are shown in the darker color (violet). All As-As bonds longer than $300 \mathrm{pm}$ are not drawn. Note the similarity between the atomic arrangements. roughly the same as the sum for four isolated As antisites (see Table I). Since $\langle 001\rangle$ is the usual growth direction of LT GaAs, it can be concluded that agglomeration of As antisites is not likely to occur during the growth process itself. Indeed, no experimental indications of this have ever been found.

While the binding energy per As antisite to the most stable clusters increases monotonically with the size of the cluster, as shown in Fig. 1, there are crucial dependencies on the geometrical configuration. Dissociating the Asantisite pair costs $200 \mathrm{meV}$. This dissociation energy increases for the most stable agglomerates from 0.32 (3 to 2 As antisite) to $1.6 \mathrm{eV}$ (6 to 5 As antisites), as shown in Fig. 3. This is the additional energy needed for enabling the growth of larger As clusters at the expense of the last smaller stable ones. At the same time the average energy gain per $\mathrm{As}_{\mathrm{Ga}}$ has increased from roughly $0.1 \mathrm{eV}$ for the $\mathrm{As}_{\mathrm{Ga}}$ pair to about $0.7 \mathrm{eV}$ for the six-atom $\mathrm{As}_{\mathrm{Ga}}$ cluster (see Fig. 1).

The energy for detachment of the last As antisite is largest for a small cluster of six As antisites $(1.6 \mathrm{eV})$. For seven As antisites, we tried different configurations but could not succeed in finding a place where one additional antisite would bind with an energy of more than $1.0 \mathrm{eV}$. For eight As antisties in the cluster, the binding situation becomes even more difficult - from the configurations we tested, only one shows a binding energy of $0.1 \mathrm{eV}$, while for the others the additional As antisites do not stick.

Agglomerates of spherical shape are not necessarily the most stable configuration for a given number of As antisites. This is especially true for smaller agglomerates, which prefer to grow on two successive $\{111\}$ planes. On these $\{111\}$ planes, the As antisites form, together with As atoms on lattice sites, configurations as in bulk As, exhibiting three short bonds of about 235-250 pm, while the distance to the next $\{111\}$ plane containing the other antisites becomes larger. In fact, this distance becomes as large as about 320 pm (see Figs. 2 and 4), which is roughly the longer bonding distance in bulk As: $311 \mathrm{pm}$.

We find - at least up to six As antisites - that the preferred growth would be on two consecutive $\{111\}$ planes, opening the possibility to have a bonding configuration similar to that in bulk As. For even larger agglomerates, we could test only a few configurations due to the vast number

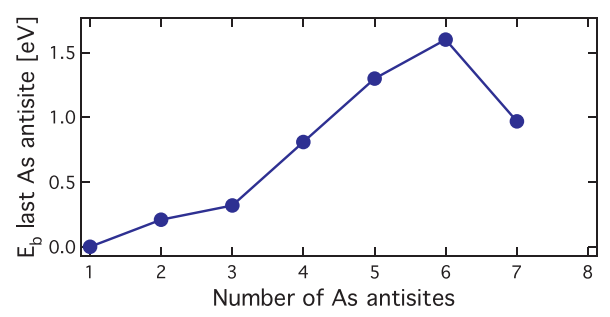

FIG. 3 (color online). Binding energy $E_{\mathrm{b}}$ for the $n$th As antisite attached to the most stable As-precipitates consisting of $n-1$ As antisites. 


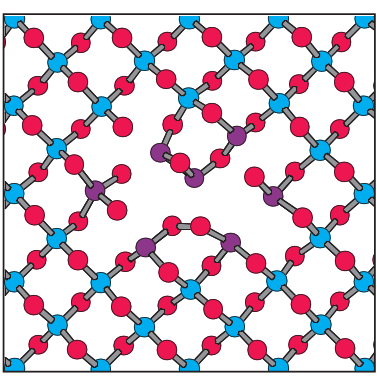

(001) plane

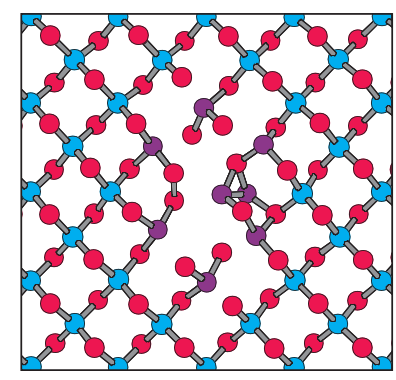

(010) plane
FIG. 4 (color online). Large agglomeration between 1.2 and $1.4 \mathrm{~nm}$ in diameter. Seventeen As antisites (darker color or violet) arranged as 5, 7, and again 5 antisites on three consecutive $\{111\}$ planes. The missing As-As bonds not drawn are longer than $300 \mathrm{pm}$.

of possibilities. Among them are two compact clusters on three $\{111\}$ planes: 15 antisites $(3,7$, and 3 antisites on each plane) and 17 antisites (5, 7, and 5 on each plane), shown in Fig. 4. We also considered one cluster having the shape of a rounded tetrahedron on three $\{111\}$ planes $(9,5,2)$. In all cases the binding energy per As antisite has increased compared to the smaller ones, especially for one As cluster similar to the tetrahedron shaped one but having 14 As antisites on two successive $\{111\}$ planes (see Table I).

Since in the case considered here even the smallest possible nucleus has locally the chemical composition of pure As as found in TEM, spinodal decomposition is unlikely as the mechanism for nucleation of precipitates.

Assuming that all excess As is already precipitated, larger agglomerates will grow with increasing temperature at the expense of smaller ones, since the binding energy for all clusters increases with their size - at least up to six As antisites. Hence, the coarsening of clusters seems to happen analogously to the macroscopic description of Ostwald ripening, as indeed found for much larger As precipitates in LT GaAs [9].

In summary, we applied a density-functional based tightbinding method to calculate formation energies of As agglomerates in $\mathrm{GaAs}$ as well as for finding the binding energies of additional As antisites to existing clusters of different sizes. In As-rich GaAs the nucleation and growth process starts on the atomic scale by rearranging atomic bonds in the energetically most favorable way. The energy gain by forming precipitates is much larger than that obtained by straining the lattice in the growth direction (7 meV per $\mathrm{As}_{\mathrm{Ga}}$ ) [13]. Hence, mobile $\mathrm{As}_{\mathrm{Ga}}$ will tend to agglomerate.

Provided there are no nucleation centers such as dislocations, it would be statistically extremely unlikely that a critical nucleus of even a small number of atoms could just randomly form. We conclude that nucleation and growth starts on the atomic level without any need for a critical nucleus size. This macroscopic description does indeed break down on an atomic scale. A necessary condition for homogeneous nucleation seems to be just a finite binding energy between two antisites.

This has many important implications far beyond this special system and semiconductors: one example is the technologically important precipitation-hardened aluminum alloys, where the understanding of the precipitation process and the subsequent growth of the precipitations is essential for designing the material properties.

We thank the Center of Scientific Computation (CSC) Helsinki for generous computing resources on the CrayT3E, as well as the NIC Jülich for providing computing resources in the project hbo21 on the T3E. T.E.M.S. acknowledges support from the Marie Curie Fund during the stay at the Helsinki University of Technology.

*Also at Laboratory of Physics, Helsinki University of Technology, Finland.

[1] The probability for randomly forming an As-antisite pair is just $\left[1-(0.99)^{8}\right] \times 0.01=7.7 \times 10^{-4}$ or $0.077 \%$.

[2] M. Kaminska et al., Appl. Phys. Lett. 54, 1881 (1989).

[3] M. Luysberg et al., J. Appl. Phys. 83, 561 (1998).

[4] Z. Liliental-Weber, K. M. Yu, J. Washburn, and D.C. Look, J. Electron. Mater. 22, 1395 (1993).

[5] X. Liu et al., Appl. Phys. Lett. 65, 3002 (1994).

[6] M. R. Melloch, N. Otsuka, J. M. Woodall, A. C. Warren, and J. L. Freeouf, Appl. Phys. Lett. 57, 1531 (1990).

[7] J. Gebauer et al., Appl. Phys. Lett. 71, 638 (1997).

[8] T. Laine, K. Saarinen, P. Hautojärvi, C. Corbel, and M. Missous, J. Appl. Phys. 86, 1888 (1999).

[9] Z. Liliental-Weber, X. W. Lin, and J. Washburn, Appl. Phys. Lett. 66, 2086 (1995).

[10] M. Bockstedte and M. Scheffler, Z. Phys. Chem. 200, 195 (1997).

[11] J. Dabrowski and J.E. Northrup, Phys. Rev. B 49, 14286 (1994).

[12] K. Tillmann, M. Luysberg, P. Specht, and E. R. Weber, Thin Solid Films 437, 74 (2003).

[13] T. E. M. Staab et al., Phys. Rev. Lett. 87, 045504 (2001).

[14] J. Dabrowski and M. Scheffler, Phys. Rev. B 40, 10391 (1989).

[15] D. J. Chadi and K. J. Chang, Phys. Rev. Lett. 60, 2187 (1988).

[16] P. Haasen, Physikalische Metallkunde (Springer Verlag, Berlin, 1984), 2nd ed.

[17] M. Haugk, J. Elsner, and Th. Frauenheim, J. Phys. Condens. Matter 9, 7305 (1997).

[18] M. Haugk, J. Elsner, Th. Heine, Th. Frauenheim, and G. Seifert, Comput. Mater. Sci. 13, 239 (1999).

[19] T.E. M. Staab, M. Haugk, Th. Frauenheim, and H.S. Leipner, Phys. Rev. Lett. 83, 5519 (1999).

[20] J.E. Northrup and S. B. Zhang, Phys. Rev. B 47, 6791 (1993).

[21] X. Liu et al., Appl. Phys. Lett. 67, 279 (1995).

[22] A. Clavierie and Z. Liliental-Weber, Philos. Mag. A 65, 981 (1992). 